\title{
Defining the impact of maternal cell contamination on the interpretation of prenatal microarray analysis
}

\author{
Allen N. Lamb, PhD'1,2, Jill A. Rosenfeld, MS1, Justine Coppinger, MS'1, Erin T. Dodge, MFA, MA ${ }^{1}$, \\ Mindy Preston Dabell, MS', Beth S. Torchia, PhD1', J. Britt Ravnan, PhD', Lisa G. Shaffer, PhD' \\ and Blake C. Ballif, PhD ${ }^{1}$
}

Purpose: To understand the ability of microarray-based comparative genomic hybridization to detect copy-number variation in the presence of maternal cell contamination.

Methods: To simulate maternal cell contamination, normal female DNA was mixed at various levels with DNA carrying known copynumber variations. Mixtures were run on a whole-genome $135 \mathrm{~K}$ oligonucleotide-based array. Data were analyzed with custom analysis software.

Results: The array and software design allowed detection of larger copy-number variations at higher levels of maternal cell contamination than smaller copy-number variations. The smallest duplications and deletions were obscured at $22-31 \%$ and $55-58 \%$ maternal cell contamination, respectively. With male fetal samples, the sex chromosome ratios started showing observable shifts at $\sim 10 \%$ maternal cell contamination.
Conclusion: As knowledge of the maternal cell contamination level aids in interpretation of array results, we recommend concurrent, independent maternal cell contamination studies for all fetal samples for accurate and timely results. With male fetal samples in our laboratory, interfering levels of maternal cell contamination can be excluded when the sex chromosome plots appear normal. Thus, reportable male microarray-based comparative genomic hybridization results may be occasionally achieved without maternal cell contamination studies. Because the effects of maternal cell contamination on microarray results are dependent on array platforms, experimental techniques, and software algorithms, each laboratory should perform its own analysis to determine acceptable levels of maternal cell contamination for its assays.

Genet Med 2012:14(11):914-921

Key Words: aCGH; maternal cell contamination; MCC; microarray; prenatal diagnosis

\section{INTRODUCTION}

Prenatal samples obtained by amniocentesis or chorionic villus sampling (CVS) are at risk of contamination by maternal cells, usually due to the presence of maternal blood or deciduas, respectively. Determining that prenatal specimens are free of significant maternal cell contamination (MCC) is important to achieve accurate results for decisions about pregnancy management.

Experience with cytogenetic analysis in many laboratories demonstrates that short-term cultures of amniocytes and chorionic villi are rarely at risk of selectively growing out large quantities of maternal cells. ${ }^{1-6}$ Therefore, when MCC is encountered in traditional cytogenetic analysis, it is usually at low levels and seldom interferes with accurate determination of the fetal karyotype. Thus, MCC testing has rarely been performed on samples undergoing standard cytogenetic analysis (reviewed in ref. 7). Experience also shows that, in general, MCC is of more concern in CVS cultures than in amniocyte cultures, due to the difficulty of successfully removing all of the maternal decidua from villi. ${ }^{1,8}$ However, when observing blood in amniocyte pellets and seeing only normal female cells on rapid aneuploidy screening by polymerase chain reaction-based methods or fluorescence in situ hybridization, some cytogenetic laboratories may place cautionary notes on reports. ${ }^{2-4,9-11}$ In contrast to traditional cytogenetic analysis, MCC analysis for prenatal molecular testing for single-gene disorders has been recommended by several genetic societies (the American College of Medical Genetics and Genomics, ${ }^{12}$ the UK Clinical Molecular Genetics Society, ${ }^{13}$ and the Canadian College of Medical Geneticists ${ }^{7,14}$ ) and the Association for Molecular Pathology $y^{15}$, although not all labs consistently follow this recommendation. ${ }^{8}$ These societies recommend that labs performing prenatal molecular testing understand how MCC may affect detection of normal and abnormal results for each assay. Recently, the Association for Molecular Pathology recommended MCC analysis for all prenatal samples undergoing molecular testing and for those undergoing molecular cytogenetic analyses such as microarraybased comparative genomic hybridization (aCGH). ${ }^{15}$

Microarray analysis is a relatively recent tool for prenatal diagnosis, with recommendations focused on using this technology for pregnancies with abnormal ultrasound findings and normal chromosome analysis. ${ }^{16}$ This algorithm usually requires

The first two authors contributed equally to this work.

${ }^{1}$ Signature Genomic Laboratories, PerkinElmer, Inc., Spokane, Washington, USA; ${ }^{2}$ Present address: ARUP Laboratories, University of Utah, Salt Lake City, Utah, USA.

Correspondence: Blake C. Ballif (blake.ballif@perkinelmer.com) 
any remaining cultures after karyotyping to be subcultured and thus increases the time in culture, which may potentially propagate any maternal cells present. This risk would generally be higher for cultured CVS than for amniocytes. ${ }^{1,8}$ For microarray analysis of direct amniocytes, the sample would be at risk of MCC due to the presence of maternal blood in the cell pellet, and for CVS the risk would be due to any maternal decidua that is not successfully identified and removed.

The ability of aCGH to detect low-level mosaicism has been documented, ${ }^{17-19}$ and it has been suggested that high levels of MCC may not impact the identification of fetal abnormalities by aCGH. ${ }^{20}$ In this study, we sought to determine the sensitivity of our prenatal microarray to MCC and the threshold of our prenatal microarray to return diagnostic results in the presence of MCC.

\section{MATERIALS AND METHODS}

\section{MCC simulation}

To better understand the risks of MCC obscuring results in prenatal aCGH testing, we performed experiments to simulate MCC by adding normal female DNA in increasing 5 or $10 \%$ increments to DNA samples, from males when possible, with known copy-number variations (CNVs). The studied CNVs, which included both duplications and deletions, and the simulated MCC levels are shown in Table 1 . The $\log _{2}$ ratios for the $\mathrm{X}$ and $\mathrm{Y}$ chromosomes were monitored in these experiments to detect the presence of any shifts that might indicate the presence of MCC.

The samples were run on a $135 \mathrm{~K}$-feature, whole-genome oligonucleotide-based microarray (SignatureChip OS version 2, custom-designed by Signature Genomics, Spokane, WA; manufactured by Roche NimbleGen, Madison, WI) as previously described. ${ }^{21}$ Data were analyzed and displayed with custom oligonucleotide aCGH analysis software (Genoglyphix, Signature Genomics). The software is set to flag segments of copy-number gain or loss involving a minimum of five probes and $\log _{2}$ of the normalized ratio of the sample:control signal intensities ( $\log _{2}$ ratio) of \pm 0.3 using a proprietary segmentation algorithm. On this array platform, segments covered by five probes are either $\sim 40 \mathrm{~kb}$, in targeted regions of the genome, or $\sim 140 \mathrm{~kb}$, in regions of backbone coverage. In addition, segments containing 200 probes with a mean $\log _{2}$ ratio of \pm 0.2 and segments containing 500 probes with a mean $\log _{2}$ ratio of \pm 0.1 are also flagged. These probe cutoffs correspond, on average, to segments that are 4.3 and $10.9 \mathrm{Mb}$, respectively.

\section{MCC detection in clinical samples}

Records for amniotic fluid and CVS samples received in our clinical laboratory for aCGH testing from July 2004 to December 2011 were reviewed for the presence of MCC. Clinical specimens were run on targeted bacterial artificial chromosome-based, whole-genome bacterial artificial chromosome-based, or whole-genome oligonucleotidebased arrays as previously described, ${ }^{6,21-25}$ depending on time of testing and clinician choice. During this period MCC testing was an optional test, with the decision to perform testing made by ordering clinicians. MCC testing was performed at ARUP Laboratories using the AmpFlSTR Indentifiler Kit (Applied Biosystems, Carlsbad, CA) or at Genzyme Genetics (Cambridge, MA) using a laboratorydeveloped, polymerase chain reaction-based assay. ARUP Laboratories reported a quantitative level of MCC, whereas

Table 1 Known copy-number variations used in the experiments to simulate MCC

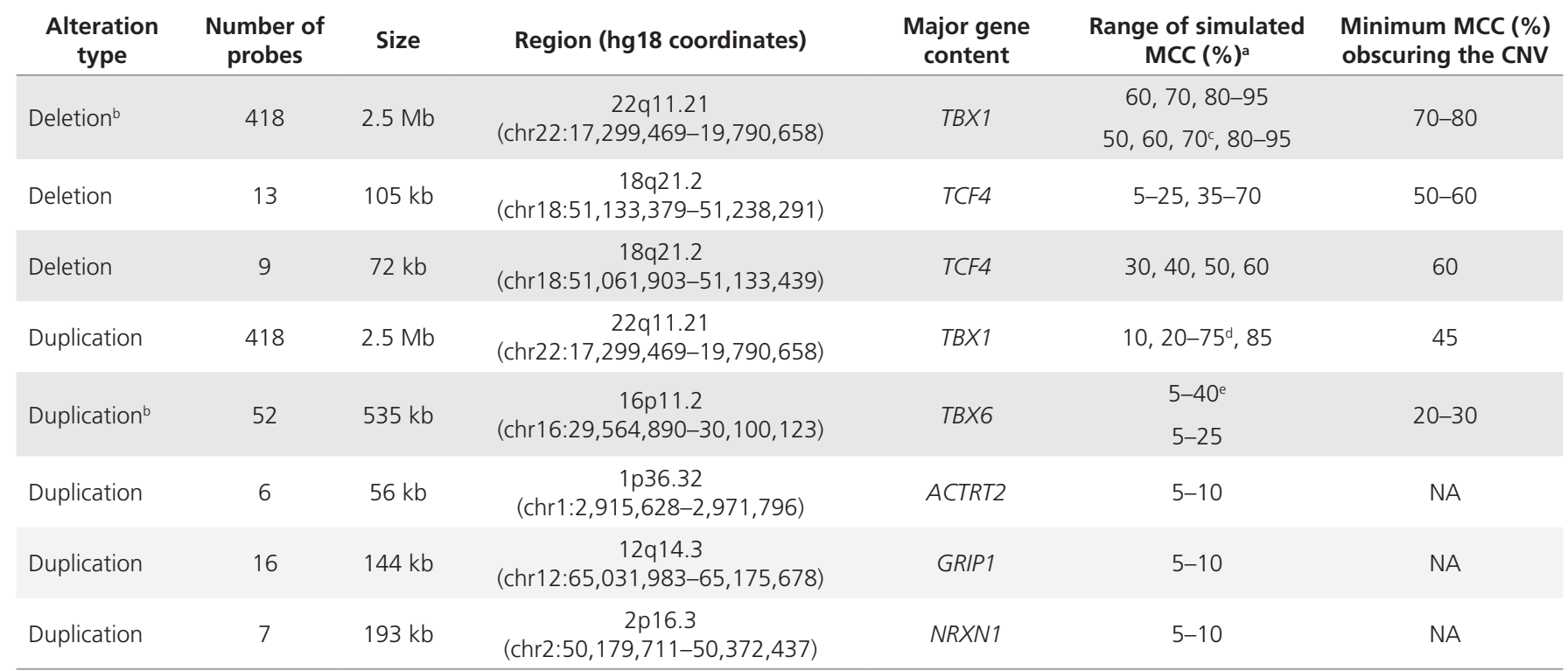

CNV, copy-number variation; MCC, maternal cell contamination; NA, not applicable.

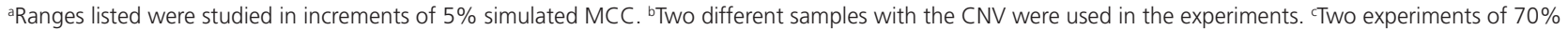

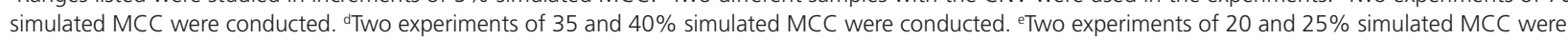
conducted. 
Genzyme Genetics reported only presence or absence of MCC.

\section{MCC simulation}

\section{RESULTS}

Preliminary results from some of these experiments have been discussed previously. ${ }^{26}$ The normalized ratios of the sample:control signal intensities in the CNV regions from the simulated MCC experiments are plotted in Figure 1a,b. Data from all simulated CNV experiments were combined to determine effects of MCC, independent of genomic region. Due to the variable $\log _{2}$ ratio cutoffs used by the software, which are dependent on the number of probes involved, larger abnormalities remain detectable at higher levels of MCC than smaller abnormalities (Figure 2). Deletions are detectable at higher levels of MCC than duplications. Using linear regression $\left(R^{2}=0.9735\right.$ for deletions and 0.7515 for duplications), we determined that, on average, the $\log _{2}$ ratios reach the -0.3 , -0.2 , and -0.1 cutoffs at $57 \%$ MCC ( $95 \%$ confidence interval (CI): $55.1-58.4 \%$ ), $72 \%$ MCC (95\% CI: 69.9-73.8\%), and $88 \%$ MCC (95\% CI: 85.5-90.7\%), respectively. For duplications, the $0.3,0.2$, and 0.1 thresholds were reached at $27 \%$ (95\% CI:
$22.3-30.8 \%), 45 \%$ (95\% CI: 40.5-51.5\%), and 63\% (95\% CI: 56.0-72.3\%) MCC, respectively (Figure 1a,b).

We also sought to understand the response of the sex chromosomes in males at lower levels of simulated MCC. Using linear regression with the normalized signal intensity ratios of the entire $\mathrm{X}$ and $\mathrm{Y}$ chromosomes (excluding the pseudoautosomal regions for the $\mathrm{Y}$ chromosome data) at $0-25 \%$ levels of female DNA $\left(R^{2}=0.8909\right.$ for $\mathrm{X}$ and 0.8028 for $\left.\mathrm{Y}\right)$, we estimate that, on average, the $\mathrm{X}$ chromosome reaches the 0.1 threshold at $10 \%$ MCC (95\% CI: 8.4-11.0\%), and the Y chromosome reaches the -0.1 threshold at $14 \%$ MCC (95\% CI: $12.0-16.0 \%$ ) (Figure $\mathbf{1 c}, \mathbf{d})$. These are conservative estimates, as they were based on the ratios of the entire $\mathrm{X}$ or $\mathrm{Y}$ chromosome, and segments of the chromosomes were frequently flagged before the ratio for the entire chromosome reached the threshold.

\section{MCC detection in clinical samples}

Our clinical experience with 2,117 prenatal specimens on which MCC testing was performed is summarized in Table 2. When samples showed MCC at levels $<20 \%$, array results were reported with the caveat that low-level mosaicism of fetal chromosomal imbalances could be masked by MCC, but nonmosaic
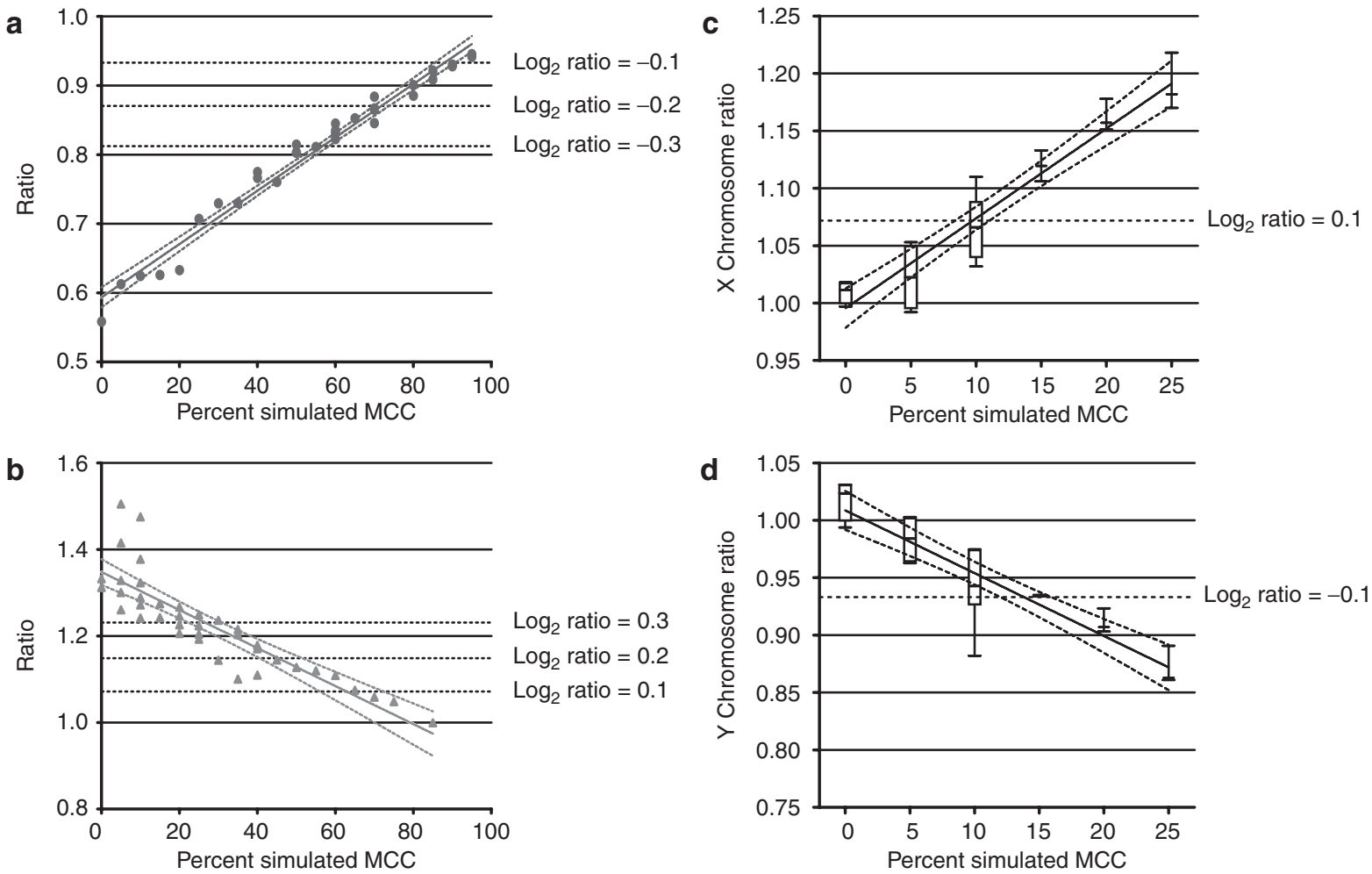

Figure 1 Normalized signal intensity ratios (sample:control) for CNVs plotted against percentage simulated MCC and box-and-whisker plots for normalized signal intensity ratios (sample:control) for sex chromosomes plotted against percentage simulated MCC. (a) Raw data (blue circles) and linear regression (blue line) for deletions. Also shown is the $95 \% \mathrm{Cl}$ for the linear regression (blue dotted lines). (b) Raw data (triangles) and linear regression (line) for duplications. Also shown is the $95 \% \mathrm{Cl}$ for the linear regression (dotted lines). Horizontal dashed lines represent the cutoffs set by the software for flagging abnormalities of various sizes (see Materials and Methods). (c) Average ratio over the whole X chromosome with linear regression (solid line) and $95 \%$ $\mathrm{Cl}$ (dashed lines). (d) Average ratio over the $\mathrm{Y}$ chromosome (excluding pseudoautosomal regions) with linear regression (solid line) and $95 \% \mathrm{Cl}$ (dashed lines). Horizontal dashed lines represent cutoffs for the software to flag ratio shifts in regions involving more than 500 probes ( $\log _{2}$ ratio of \pm 0.1 ). $\mathrm{Cl}$, confidence interval; CNV, copy-number variation; MCC, maternal cell contamination. 


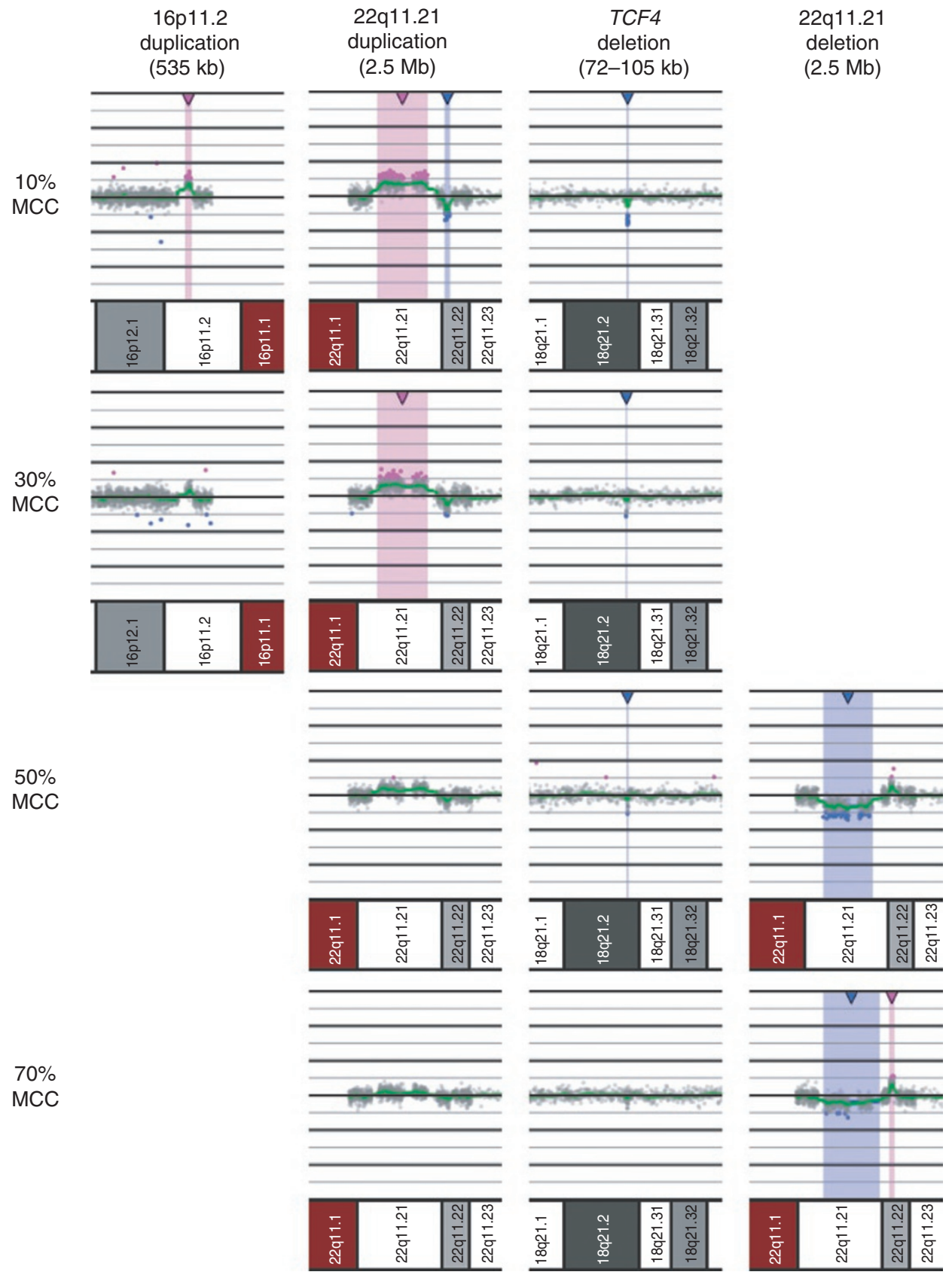

Figure 2 Microarray results from simulated MCC experiments. CNVs being tested and levels of simulated MCC are as labeled; additional information on the CNVs studied is in Table 1. The smallest duplications (pink-shaded regions) are first to be obscured by increasing levels of MCC, and the largest deletions (blue-shaded regions) are the last to be obscured. In some cases, the CNVs may be visually detectable, even when the software has not flagged them, such as the 22q11.21 duplication at 50\% MCC. Certain case examples show secondary, benign copy-number changes; the 22 q11.21 duplication example has a more distal deletion, and in the 22q11.21 deletion example, the normal female DNA has a more distal duplication that becomes apparent at higher levels of MCC. CNV, copy-number variation; MCC, maternal cell contamination.

abnormalities were not detected. Any samples showing higher levels of MCC were reported as having uninformative array results. We had seven cases in which an abnormality was present and MCC testing showed contamination: in two cases, MCC was $>20 \%$, and the abnormality was carried by the mother (Figure 3a,b); the other five cases had CNVs carried by the fetus and an MCC level $<5 \%$. In three of these cases, the mother did not carry the CNV. In one of the two cases with high levels of MCC and a maternal CNV detected by the array, a second sample from an independent noncontaminated culture confirmed that the fetus did not carry the duplication. In addition, in one male fetal sample without MCC studies performed, array results were suggestive of MCC due to shifts in the $\log _{2}$ ratios of the sex chromosomes, and a $230-\mathrm{kb}$ clinically significant deletion including FOXF1 was also present (Figure 3c). In this case, fluorescence in situ hybridization studies demonstrated that 
Table 2 Detection of MCC in our experience of clinical prenatal specimens received from July 2004 through December 2011

\begin{tabular}{|c|c|c|c|c|c|c|c|}
\hline \multirow[b]{2}{*}{ Fetal sex } & \multicolumn{2}{|c|}{$\begin{array}{c}\text { Total cases with MCC } \\
\text { testing }\end{array}$} & \multicolumn{2}{|c|}{$\begin{array}{l}\text { Cases with low-level MCC not } \\
\text { affecting results }(\leq 20 \%)\end{array}$} & \multicolumn{3}{|c|}{$\begin{array}{c}\text { Cases with high-level MCC }(>20 \%) \text { resulting } \\
\text { in uninformative aCGH results }\end{array}$} \\
\hline & Male & Female & Male & Female & Male & Female & Total (\%) \\
\hline Cultured amniocytes & 679 & 707 & 3 & 5 & 2 & 2 & $4(0.3)^{a}$ \\
\hline Direct amniocytes & 62 & 76 & 3 & 1 & 0 & 0 & $0(0.0)$ \\
\hline Cultured CVS & 262 & 275 & 9 & 5 & 2 & 5 & $7(1.3)^{b}$ \\
\hline Direct CVS & 34 & 22 & 1 & 3 & 0 & 0 & $0(0.0)$ \\
\hline
\end{tabular}

A portion of these data was previously described. ${ }^{26} \mathrm{aCGH}$, array-based comparative genomic hybridization; CVS, chorionic villi sample; MCC, maternal cell contamination.

ane additional sample from a pregnancy with a female fetus had significant MCC in the first sample tested, but DNA isolated from a second culture had no MCC present (Figure 3a,b). Another sample from a pregnancy with a male fetus (46,XY karyotype) without MCC studies performed had array results consistent with MCC, and fluorescence in situ hybridization showed $23 \%$ of cells to have an XX sex chromosome complement; a clinically significant deletion was reported in this case (Figure 3c). ${ }^{b}$ Five additional samples without MCC studies performed and from pregnancies with male fetuses with previous karyotype studies showing $46, X Y$ had array results consistent with a majority of cells being $46, X X$, leading to suspected MCC and uninformative array results reported.

$23 \%$ of cells had an XX sex chromosome complement, whereas the deletion was present exclusively in the male cells. Therefore, although MCC was suspected at a $>20 \%$ level, it was possible to report a significant abnormality in this case.

In clinical samples from male fetuses in which MCC was confirmed through independent MCC testing, the sex chromosomes were sometimes flagged as abnormal by the software. Some of these cases were run on earlier, bacterial artificial chromosome-based arrays using sex-mismatched controls; consequently, sex chromosome $\log _{2}$ ratios normally deviated from zero, and the software was not set to flag these regions. Of the 15 cases with MCC that were male fetuses tested on oligonucleotide-based platforms with sex-matched controls, 10 did not have the sex chromosomes flagged: 9 with MCC at levels $<5 \%$ and 1 with $6-10 \%$ MCC. The other five had the sex chromosomes flagged; the lowest level of MCC among these cases as determined by independent testing was $5 \%$. In this case most of the $\mathrm{X}$ chromosome was flagged; the average $\log _{2}$ ratio over the entire chromosome was 0.09 (Figure 3d). Five additional samples from pregnancies with male fetuses (46,XY karyotypes) run on oligonucleotide arrays had MCC suspected based on the flagged, abnormal $\mathrm{X}$ and $\mathrm{Y}$ chromosome ratios. However, in these cases independent MCC testing was not performed, so levels of MCC are unknown.

\section{DISCUSSION}

Every test used for prenatal diagnosis may have a different sensitivity threshold for MCC and should be studied for the effects of MCC. For aCGH, as expected based on the predicted $\log _{2}$ ratio (sample:control) for a deletion $\left[\log _{2}(1: 2)=-1\right]$ relative to the predicted $\log _{2}$ ratio for a duplication $\left[\log _{2}(3: 2)=0.58\right]$, duplications become obscured at a lower level of MCC than similarly sized deletions (Figures $\mathbf{1 a}, \mathbf{b}$ and 2 ). Our results show that for our prenatal oligonucleotide-based microarrays and accompanying display software, MCC obscures small $(<\sim 4.3$ $\mathrm{Mb}$ ) fetal deletions starting at 55-58\% MCC and small fetal duplications starting at $22-31 \%$ MCC. Due to the less strict criteria used by the software for identifying larger CNVs, the largest (> 10.9 Mb) deletions will remain detectable until $86-91 \%$
MCC and duplications will remain detectable until 56\%-72\% MCC (Figure 1a,b). For all abnormalities, visual ascertainment of the CNVs, when not flagged by the software, may be possible at higher levels of MCC, although the ability to do so will be limited by the overall quality of the array data (Figure 2).

With a female fetus tested by aCGH, no difference in the sex chromosome complement exists to suggest the presence of MCC, so MCC testing is required to be confident that the results are reflective of the fetal DNA. For male fetal samples with MCC, however, the X chromosome showed an apparent mosaic gain and was flagged by the software starting at about $10 \%$ MCC. Because our data demonstrate that even small duplications are detectable at 10\% MCC (Figure 2) and because a male result not showing a shifted $\mathrm{X}$ chromosome plot would be expected to have MCC of $<10 \%$, it is possible to be confident that a normal male aCGH result has excluded nonmosaic CNVs without the need for MCC studies, provided that the quality of the array data is optimal. However, when array results for a male fetus are suggestive of MCC due to shifted sex chromosome plots, MCC testing would be needed to confirm that MCC is the explanation for the sex chromosome shifts, as well as to determine the level of MCC present to aid in the final interpretation of the array results. Therefore, for accurate and timely results, we recommend MCC testing be undertaken concurrently with prenatal testing even on a male fetus.

If independent MCC testing is not performed with a male fetal sample with normal sex chromosome plots, it is still possible that cryptic MCC $<10 \%$ could mask a mosaic abnormality in the fetus. However, such low-level MCC would only slightly alter the ability of the array to detect fetal mosaicism. Assuming that oligonucleotide-based aCGH can reliably detect mosaicism starting at a $30 \%$ level, ${ }^{19,27}$ then with $10 \%$ MCC, the reliable detection level for fetal mosaicism only increases to $33 \%$. Of note, the simulated MCC experiments presented here could also be viewed as simulated mosaicism experiments (when viewed inversely), and our data reveal that it may not be appropriate to use a single cutoff for detection of mosaicism; as shown, larger abnormalities, as well as deletions, remain detectable by the aCGH software at a much lower level of mosaicism 
a

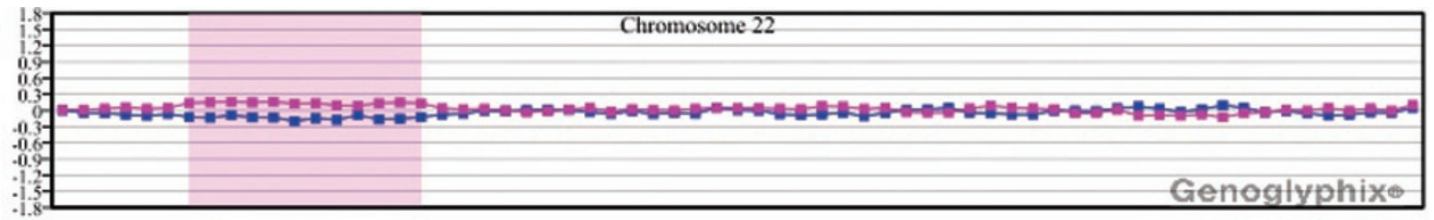

b

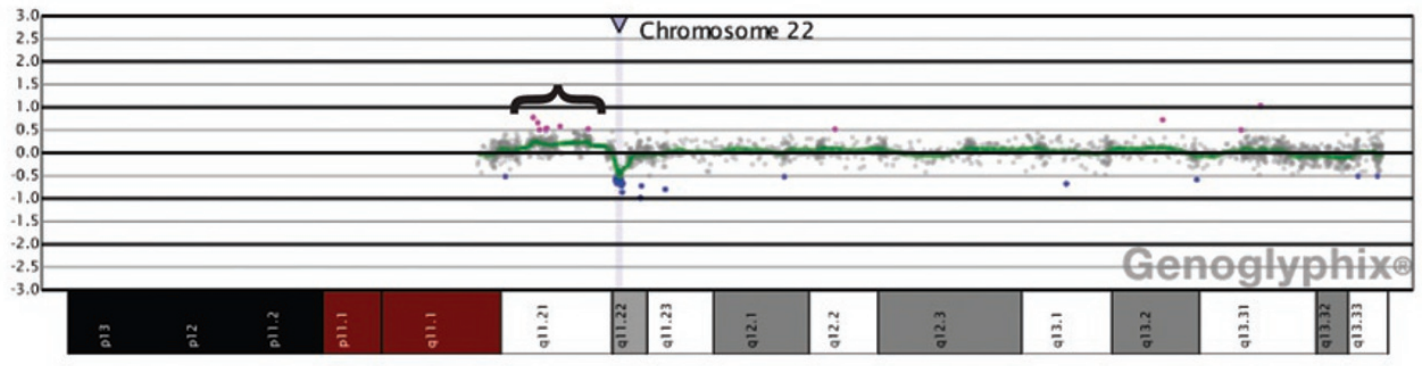

c

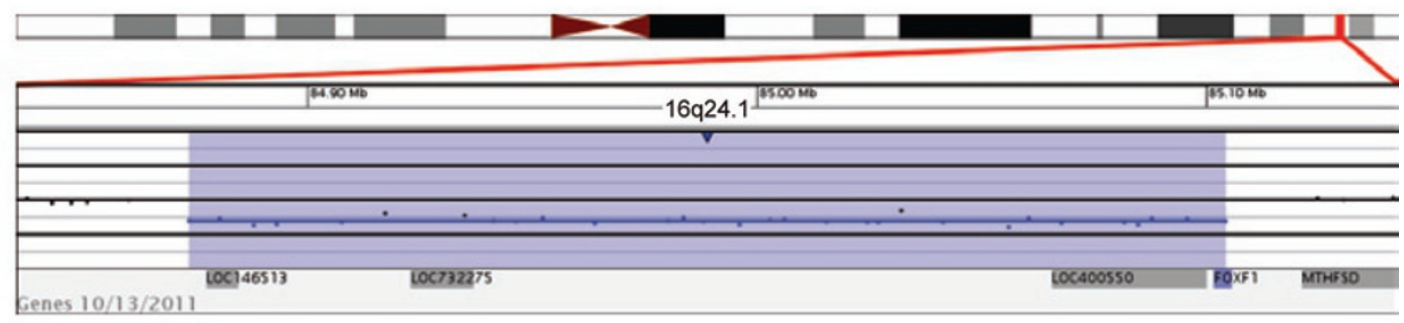

d

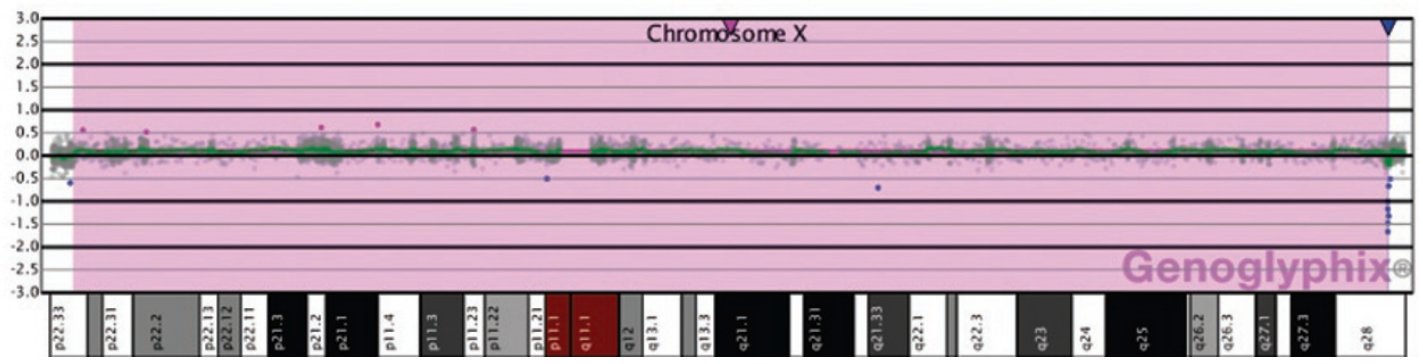

Figure 3 Case examples of MCC. (a,b) Chromosome 22 microarray results on a cultured amniotic fluid specimen from a female fetus tested due to family history of 1q21.1 microdeletion and 22q11.21 microduplication demonstrated a mosaic gain of 22q11.21, which was identified using a BAC-based array with dye-swap experiments (a) but was not flagged by the software when run on an oligonucleotide-based array (b; bracket). FISH on the same cultured fetal specimen did not confirm the microduplication. DNA-based MCC testing showed the presence of MCC at $30 \%$ maternal cells. Microarray analysis was performed on a second independent culture from the same direct amniotic fluid specimen, and results were normal female (data not shown); MCC testing was negative on this sample. The microduplication was confirmed to be in the mother, not the fetus. (c) Microarray results on a cultured amniotic fluid specimen from a male fetus $(46, X Y$ karyotype) tested due to ultrasound findings of bilateral pyelectasis and megacystis demonstrated a $230-\mathrm{kb}$ deletion on $16 \mathrm{q} 24.1$ including FOXF1. The $X$ and $Y$ chromosome array results in this experiment showed shifts consistent with MCC (data not shown). FISH showed a $Y$ chromosome in 59/77 cells (77\%), and only the Y-containing cells also had the 16q24.1 deletion. The deletion was reported as clinically significant, and the report included a caveat that other small CNVs could have been masked by suspected MCC. (d) Chromosome X microarray results on a cultured CVS sample from a male fetus referred for abnormal maternal serum screen and increased nuchal translucency showed an average $\log _{2}$ ratio of 0.09 over the whole chromosome; the software flagged the segment reaching a $0.1 \log _{2}$ ratio. Interphase FISH showed 2/50 cells to have an XX chromosome complement, and DNA-based MCC testing showed contamination at $5 \%$ maternal cells. No other significant abnormalities were detected on array, and the result was reported as normal male with the presence of low-level MCC, which could mask fetal mosaic abnormalities. BAC, bacterial artificial chromosome; CNV, copy-number variation; CVS, chorionic villus sampling; FISH, fluorescence in situ hybridization; MCC, maternal cell contamination.

than do small abnormalities and duplications (Figures 1a,b and 2). Previous studies have suggested that the ability of the array to detect mosaicism may vary according to the size of the aneuploid segment, ${ }^{19}$ and our experiments address these questions for CNVs smaller than previously considered. Even if we assume that the test has a limitation of reliable mosaicism detection of at least $73 \%$ abnormal cells with small duplications (on the basis of the experiments presented here), then the presence of $10 \%$ MCC would change this reliable detection level to $81 \%$ fetal mosaicism. Therefore, MCC testing on a sample with normal male array results would only provide a slight change in the detection threshold of mosaicism.

Careful consideration needs to be made when apparently mosaic results are present in a prenatal sample, as MCC, true fetal mosaicism, confined placental mosaicism (in a CVS sample), or even the presence of triploidy can cause such 
array patterns. In addition, studies have suggested that mosaicism may be more common than appreciated before the use of microarrays for the detection of chromosomal copy losses and gains. ${ }^{17-19,27}$ As a general rule, for a male fetal sample, a result showing a mosaic CNV without a detectable change in the $\mathrm{X}$ and $\mathrm{Y}$ chromosomes would indicate fetal mosaicism or confined placental mosaicism. If shifts in the $\mathrm{X}$ or Y plots are present with a male fetal sample, this may indicate MCC, and any mosaic-appearing $\mathrm{CNV}$ may represent a fetal or maternal change. In addition, although a 47,XXY karyotype will appear as a gain of X without a loss of $\mathrm{Y}$, a $69, \mathrm{XXY}$ karyotype may look like MCC, with a mosaic-appearing gain of the $\mathrm{X}$ and a mosaicappearing loss of the $\mathrm{Y}^{28}$ For a female fetal sample, a result suggestive of multiple cell lines could indicate MCC with either the fetus or the mother carrying the CNV, mosaicism of fetal cells, or confined placental mosaicism; it could also mean any combination of the three. Due to these multiple possibilities in both male and female samples, when an apparent mosaic CNV is detected by microarray, testing for MCC and additional followup testing to visualize the abnormality through fluorescence in situ hybridization or karyotyping are required to obtain an accurate diagnostic result. Particularly concerning is the potential for false positives, as illustrated by the case in this study that showed a mosaic CNV that was carried by the mother and not the fetus (Figure 3a,b).

In our laboratory, to take a conservative approach in the absence of an abnormal CNV detected by aCGH, we report fetal samples with MCC $>20 \%$ as having an inconclusive/uninformative microarray result. Nonmosaic deletions and larger duplications may be excluded when MCC levels are $20 \%$ or higher; however, clinically significant small duplications may not. Using this $20 \%$ cutoff, we have experienced a rate of significant MCC in $0.3 \%$ of cultured amniocentesis samples and $1.3 \%$ of cultured CVS samples (Table 2). Although we have not had any MCC interfering with reporting of results in direct amniocyte or direct CVS samples, direct samples arousing suspicion of MCC upon receipt (i.e., blood in an amniocyte cell pellet) were cultured before running aCGH. Because of this selection bias and our small numbers, it is not possible to infer the frequency of MCC in these direct samples.

Although we used oligonucleotide-based microarrays in our experiments, some of our findings may be generalized to other types of arrays, with some notable differences. For example, because some single-nucleotide polymorphism-based arrays provide not only data on copy number but also genotypic data, the array data should be able to indicate possible MCC regardless of fetal sex and differentiate it from fetal mosaicism based on altered genotype frequencies. Single-nucleotide polymorphism arrays may be as sensitive, or more sensitive, to MCC than CGH-based arrays, as one study showed that chimerism at a $5 \%$ level could be detected. ${ }^{29}$ Research has shown that singlenucleotide polymorphism arrays may be able to detect wholechromosome mosaicism at levels as low as $5-7 \%,{ }^{29,30}$ although smaller CNVs have not been formally evaluated. ${ }^{29}$ Determining how undetected low-level MCC would alter the ability of the single-nucleotide polymorphism array to detect subtle abnormalities such as small deletions or duplications or low-level mosaicism would be important for each practicing laboratory to determine.

Given the critical nature of prenatal samples and the possibility of medium to high levels of MCC obscuring fetal abnormalities on microarray testing, it is appropriate to conduct MCC studies concurrently with all microarray studies, consistent with the recommendations of the recent Association for Molecular Pathology report. ${ }^{15}$ If MCC studies pose a challenge (e.g., financially or due to a lack of a maternal sample), aCGH results from a male fetus may sometimes still be interpreted, as data from the array experiment can be used to exclude interfering levels of MCC. For the most accurate interpretation of how MCC may affect microarray results, the independent methodology used to confirm the presence of MCC should be quantitative. If MCC is present above the level at which it could obscure results (22-31\% in our experiments), getting fully informative results would require rerunning the microarray using DNA extracted from a different culture or new fetal specimen. When a sample has MCC at a level lower than what would obscure an abnormal result $(<\sim 20 \%)$, repeating the analysis would give a slight increase in the ability to detect fetal mosaicism, and this should be described when reporting results for samples with low-level MCC. Finally, the way that MCC affects microarray results is highly dependent on array platform, experimental techniques, software algorithms, and quality of the microarray data, so it is critical that each laboratory perform its own analysis to determine acceptable levels of MCC for all prenatal assays offered. As array platforms increase in the density of coverage, with some including dense exonic-level coverage for an increasing number of genes, some very small abnormalities, which are most easily obscured by low levels of MCC, could be of clinical concern. Therefore, MCC studies should continue to be an integral part of prenatal microarray analysis.

\section{ACKNOWLEDGMENTS}

We thank Emily Hall for her assistance in carrying out these experiments and A. Michelle Caldwell for her editorial assistance.

\section{DISCLOSURE}

The authors are current or former employees of Signature Genomic Laboratories, PerkinElmer. A.N.L., J.C., J.B.R., L.G.S., and B.C.B. own stock in PerkinElmer. This study was funded by Signature Genomic Laboratories.

\section{REFERENCES}

1. Ledbetter DH, Zachary JM, Simpson JL, et al. Cytogenetic results from the U.S. Collaborative Study on CVS. Prenat Diagn 1992;12:317-345.

2. Ward BE, Gersen SL, Carelli MP, et al. Rapid prenatal diagnosis of chromosomal aneuploidies by fluorescence in situ hybridization: clinical experience with 4,500 specimens. Am J Hum Genet 1993;52:854-865.

3. Mann K, Donaghue C, Fox SP, Docherty Z, Ogilvie CM. Strategies for the rapid prenatal diagnosis of chromosome aneuploidy. Eur J Hum Genet 2004;12:907-915.

4. Stojilkovic-Mikic T, Mann K, Docherty Z, Mackie Ogilvie C. Maternal cell contamination of prenatal samples assessed by QF-PCR genotyping. Prenat Diagn 2005;25:79-83. 
5. Van den Veyver IB, Patel A, Shaw CA, et al. Clinical use of array comparative genomic hybridization $(\mathrm{aCGH})$ for prenatal diagnosis in 300 cases. Prenat Diagn 2009;29:29-39.

6. Coppinger J, Alliman S, Lamb AN, Torchia BS, Bejjani BA, Shaffer LG Whole-genome microarray analysis in prenatal specimens identifies clinically significant chromosome alterations without increase in results of unclear significance compared to targeted microarray. Prenat Diagn 2009;29:1156-1166.

7. Winsor EJ, Akoury H, Chitayat D, Steele L, Stockley TL. The role of molecular microsatellite identity testing to detect sampling errors in prenatal diagnosis. Prenat Diagn 2010;30:746-752.

8. Schrijver I, Cherny SC, Zehnder JL. Testing for maternal cell contamination in prenatal samples: a comprehensive survey of current diagnostic practices in 35 molecular diagnostic laboratories. J Mol Diagn 2007;9:394-400.

9. Smith GW, Graham CA, Nevin J, Nevin NC. Detection of maternal cell contamination in amniotic fluid cell cultures using fluorescent labelled microsatellites. J Med Genet 1995;32:61-64.

10. Frederickson RM, Wang HS, Surh LC. Some caveats in PCR-based prenatal diagnosis on direct amniotic fluid versus cultured amniocytes. Prenat Diagn 1999;19:113-117.

11. Van Opstal D, Boter M, de Jong D, et al. Rapid aneuploidy detection with multiplex ligation-dependent probe amplification: a prospective study of 4000 amniotic fluid samples. Eur J Hum Genet 2009;17:112-121.

12. ACMG. American College of Medical Genetics: Standards and Guidelines for Clinical Genetics Laboratories. 2008. http://www. acmg.net/AM/Template.cfm?Section=Laboratory_Standards_and_ Guidelines\&Template $=/ C M /$ ContentDisplay.$c f m \& C$ ontentID $=5131$. Accessed 6 March 2011.

13. CMGS. Practice Guidelines for the Testing for Maternal Cell Contamination (MCC) in Prenatal Samples for Molecular Studies. [PDF]. 2008. http:// www.cmgs.org/BPGs/pdfs\%20current\%20bpgs/MCC_08.pdf. Accessed 16 March 2011

14. CCMG. CCMG Molecular Genetics Guidelines. CCMG Molecular Genetics Committee: Ottawa, Canada, 2006:9.

15. Nagan N, Faulkner NE, Curtis C, Schrijver I; MCC Guidelines Working Group of the Association for Molecular Pathology Clininical Practice Committee. Laboratory guidelines for detection, interpretation, and reporting of maternal cell contamination in prenatal analyses a report of the association for molecular pathology. J Mol Diagn 2011;13:7-11.

16. ACOG Committee Opinion No. 446. Array comparative genomic hybridization in prenatal diagnosis. Obstet Gynecol 2009;114: 1161-1163.
17. Ballif BC, Rorem EA, Sundin K, et al. Detection of low-level mosaicism by array $\mathrm{CGH}$ in routine diagnostic specimens. Am J Med Genet $A$ 2006;140:2757-2767.

18. Cheung SW, Shaw CA, Scott DA, et al. Microarray-based CGH detects chromosomal mosaicism not revealed by conventional cytogenetics. Am J Med Genet A 2007;143A:1679-1686

19. Scott SA, Cohen N, Brandt T, Toruner G, Desnick RJ, Edelmann L. Detection of low-level mosaicism and placental mosaicism by oligonucleotide array comparative genomic hybridization. Genet Med 2010;12:85-92.

20. Shaffer LG, Bui TH. Molecular cytogenetic and rapid aneuploidy detection methods in prenatal diagnosis. Am J Med Genet C Semin Med Genet 2007;145C:87-98.

21. Duker AL, Ballif BC, Bawle EV, et al. Paternally inherited microdeletion at $15 q 11.2$ confirms a significant role for the SNORD116 C/D box snoRNA cluster in Prader-Willi syndrome. Eur J Hum Genet 2010;18:1196-1201.

22. Shaffer LG, Dabell MP, Rosenfeld JA, et al. Referral patterns for microarray testing in prenatal diagnosis. Prenat Diagn 2012;32:344-350.

23. Ballif $B C$, Theisen A, Coppinger J, et al. Expanding the clinical phenotype of the 3q29 microdeletion syndrome and characterization of the reciprocal microduplication. Mol Cytogenet 2008;1:8.

24. Ballif BC, Theisen A, McDonald-McGinn DM, et al. Identification of a previously unrecognized microdeletion syndrome of $16 q 11.2 q 12.2$. Clin Genet 2008;74:469-475.

25. Bejjani BA, Saleki R, Ballif BC, et al. Use of targeted array-based CGH for the clinical diagnosis of chromosomal imbalance: is less more? Am J Med Genet A 2005; 134:259-267.

26. Lamb AN. Laboratory aspects of prenatal microarray analysis. Clin Lab Med 2011;31:615-30, ix.

27. Neill NJ, Torchia BS, Bejjani BA, Shaffer LG, Ballif BC. Comparative analysis of copy number detection by whole-genome BAC and oligonucleotide array CGH. Mol Cytogenet 2010;3:11.

28. Ballif BC, Kashork CD, Saleki $R$, et al. Detecting sex chromosome anomalies and common triploidies in products of conception by arraybased comparative genomic hybridization. Prenat Diagn 2006;26: 333-339.

29. Bruno DL, White SM, Ganesamoorthy D, et al. Pathogenic aberrations revealed exclusively by single nucleotide polymorphism (SNP) genotyping data in 5000 samples tested by molecular karyotyping. J Med Genet 2011;48:831-839.

30. Conlin LK, Thiel BD, Bonnemann CG, et al. Mechanisms of mosaicism, chimerism and uniparental disomy identified by single nucleotide polymorphism array analysis. Hum Mol Genet 2010;19:1263-1275. 